\title{
Spontaneous emission in two-dimensional photonic crystal microcavities
}

\section{Søndergaard, Thomas}

\section{Published in:}

I E E E Journal of Quantum Electronics

Link to article, DOI:

$10.1109 / 3.831020$

Publication date:

2000

Document Version

Publisher's PDF, also known as Version of record

Link back to DTU Orbit

Citation (APA):

Søndergaard, T. (2000). Spontaneous emission in two-dimensional photonic crystal microcavities. I E E E Journal of Quantum Electronics, 36(4), 450-457. https://doi.org/10.1109/3.831020

\section{General rights}

Copyright and moral rights for the publications made accessible in the public portal are retained by the authors and/or other copyright owners and it is a condition of accessing publications that users recognise and abide by the legal requirements associated with these rights.

- Users may download and print one copy of any publication from the public portal for the purpose of private study or research.

- You may not further distribute the material or use it for any profit-making activity or commercial gain

- You may freely distribute the URL identifying the publication in the public portal

If you believe that this document breaches copyright please contact us providing details, and we will remove access to the work immediately and investigate your claim. 


\title{
Spontaneous Emission in Two-Dimensional Photonic Crystal Microcavities
}

\author{
Thomas Søndergaard
}

\begin{abstract}
The properties of the radiation field in a two-dimensional photonic crystal with and without a microcavity introduced are investigated through the concept of the position-dependent photon density of states. The position-dependent rate of spontaneous radiative decay for a two-level atom with random orientation is deduced from the photon density of states using the Fermi Golden Rule.
\end{abstract}

Index Terms-Integrated optics, microcavity, photonic bandgap, photonic crystal, spontaneous emission.

\section{INTRODUCTION}

$\mathrm{N}$ RECENT years, it has been accepted that the rate of spontaneous radiative decay for an atom may be controlled by modifying the properties of the radiation field [1]-[8]. By surrounding an excited atom with a structure, where no electromagnetic mode is available at the atomic transition frequency, spontaneous emission may be inhibited [1], [3]. If, on the other hand, the atom is placed in a cavity with a resonance at the atomic transition frequency, spontaneous emission may be significantly enhanced [2]. In semiconductor laser technology, losses due to spontaneous emission represent a fundamental limit on performance. By inhibiting spontaneous emission into modes other than the lasing mode, the threshold of future lasers may be reduced to zero [1]. A clear demonstration of suppression of spontaneous emission in the submillimeter regime of wavelengths has been reported in [3], where two parallel conducting planes were used to eliminate the electromagnetic modes at the transition frequency for atoms in a Rydberg state. At optical frequencies, however, metallic structures will no longer resemble ideal conductors due to dissipation. A promising alternative to metallic structures for control of matter-light interactions at optical frequencies is the new class of periodic dielectric structures, known as photonic crystals or photonic bandgap structures [9], [10]. These structures are characterized by having one or more frequency intervals, photonic bandgaps, where propagation of electromagnetic waves in one or more dimensions is inhibited. A three-dimensional (3-D) periodic dielectric structure may inhibit propagation of electromagnetic waves in all three dimensions for a certain frequency interval. In this case, there are no electromagnetic modes available within this frequency interval, and spontaneous emission may be rigorously for-

Manuscript received July 26, 1999; revised October 18, 1999. This work was supported by the Danish Technical Research Council under the THOR (Technology by Highly Oriented Research) program.

The author is with the Research Center COM, Technical University of Denmark, DK-2800 Lyngby, Denmark.

Publisher Item Identifier S 0018-9197(00)02700-7.

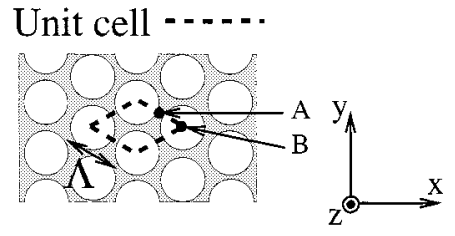

(a)

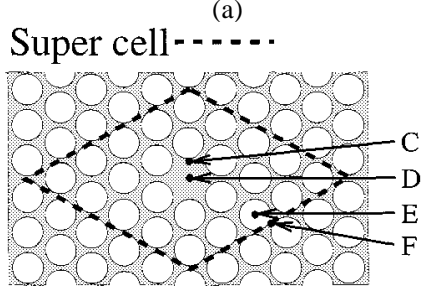

(b)

Fig. 1. (a) Triangular-hole photonic crystal with circular air holes arranged on a triangular lattice in a dielectric background with a dielectric constant of 13 . The volume fraction of the air holes is 0.625 . (b) The same structure where a microcavity has been introduced by removing a single air hole.

bidden [1]. Recently, 3-D periodic structures with photonic bandgaps at optical and near-infrared wavelengths have been successfully demonstrated [11], [12]. The fabrication of 3-D periodic structures with bandgaps at optical frequencies is, however, very difficult by today's technology. Three-dimensional periodic structures are not considered in this paper. In this paper, we will concentrate on spontaneous emission rate alteration in the two-dimensional (2-D) structures shown in Figs. 1(a) and (b). Fig. 1(a) shows a 2-D photonic crystal, where circular air holes are arranged on a triangular lattice in a dielectric background with a dielectric constant of 13. The volume fraction of the air holes is 0.625 . The photonic crystal is characterized by a frequency interval, which will be referred to as an in-plane photonic bandgap, where the propagation of electromagnetic waves in the $x y$ plane is forbidden. Fig. 1(b) shows a photonic crystal, where a defect has been introduced by removing a single air hole in the otherwise periodic structure. This particular defect has the function of a microcavity, where a few electromagnetic modes are strongly localized. Recent theoretical and experimental results for a 2-D photonic crystal microcavity may be found in [13]-[15].

In Fig. 1, a number of positions have been labeled A-F. The rate of spontaneous emission will be considered later for these positions.

Previous work on spontaneous emission in 2-D photonic crystals, where position dependence of the emitter was not taken into account, may be found in [16] and [17]. In general, the rate of spontaneous emission may depend significantly on the position of the emitter in any structure, where the dimensions are compa- 
rable to the emission wavelength of concern. Therefore, position dependence must be included for accurate modeling of spontaneous emission in photonic crystals and microcavities.

In this paper, we will model spontaneous emission rate alteration through the concept of the position-dependent photon density of states using plane-wave expansion theory. For the case of free space, the position-independent photon density of states is well known [18], and, for a two-level atom in free space, the rate of spontaneous emission may be simply related to the position-independent photon density of states. This is, however, not the case for an atom placed in a photonic crystal, where position dependence must be taken into account. Therefore, we introduce in this paper the position-dependent photon density of states, which is obtained from the position-independent photon density of states by weighting the contribution from each electromagnetic state by the position-dependent amplitude of the electric field squared. With this modification, spontaneous emission can be simply related to the now position-dependent photon density of states.

Previous theoretical calculations of the position-dependent photon density of states in photonic crystals may be found in [19] and [20]. In order to evaluate the enhancement or suppression of spontaneous emission from an excited two-level system in a photonic crystal, the position-dependent photon density of states must be compared to a proper reference. For the structures considered in this paper, where the active medium is assumed to be placed in the high-index material, a proper reference is the photon density of states in a homogeneous dielectric with the same dielectric constant as the high-index material. The first result for spontaneous emission rate alteration for a photonic crystal using this method may be found in [19]. For microcavity structures based on introducing a defect into a photonic crystal, it is important to understand spontaneous emission for an active medium close to the defect. A model for spontaneous emission near defects in photonic crystals must take into account not only spontaneous emission into the cavity modes but also spontaneous emission into a continuum of modes that are not localized to the cavity. Modeling the position-dependent photon density of states near defects in 2-D photonic crystals is a considerably more demanding numerical task compared to modeling photonic crystals. Therefore, the calculations of the density of states presented in this paper are based on a more powerful plane-wave expansion method for large numerical problems [21], [22] compared to the plane-wave expansion method [23] used for calculating the density of states for photonic crystals in [20].

The paper is organized in the following way. In Section II, a model for spontaneous emission in photonic crystals and photonic crystal microcavities is presented. In Section III, numerical results for the photon density of states in photonic crystals and photonic crystal microcavities are given. Strong peaks in the photon density of states are related to a few strongly localized cavity modes. Conclusions are given in Section IV.

\section{Model FOR SPONTANEOUS EMISSION IN 2-D PHOTONIC CRYSTAL MicRocAVITIES}

In this section, we present a model based on the Fermi Golden rule [24] for the position-dependent rate of spontaneous emis- sion for a two-level atom with random orientation. The atom is treated as an electric dipole with dipole operator $\mathbf{p}$. The firstorder interaction Hamiltonian for the interaction between the atom and the radiation field is in the space representation given by

$$
H_{I}=-\mathbf{p} \cdot \mathbf{E}
$$

where $\mathbf{E}$ is the electric field operator. In general, the classical electric field may be written as a sum of orthogonal eigenfunctions $\mathbf{e}(s, \mathbf{r})$, where the eigenfunctions denoted $s$ are solutions to the complex Maxwell equations [25]. In the Schrödinger picture, the electric field operator can be written

$$
\mathbf{E}(\mathbf{r})=-\frac{i}{\sqrt{2}} \sum_{s}\left[a_{s}-a_{s}^{\dagger}\right] \mathbf{e}(s, \mathbf{r})
$$

where $a_{s}$ and $a_{s}^{\dagger}$ are the creation and annihilation operators for modes $s$ [24]. The angular frequency of mode $s$ is denoted $\omega_{s}$. It is clear from (1) and (2) that the interaction Hamiltonian may be altered by modifying the properties of the radiation field. By requiring

$$
\int \varepsilon_{r}(\mathbf{r}) \mathbf{e}(s, \mathbf{r})^{*} \cdot \mathbf{e}\left(s^{\prime}, \mathbf{r}\right) d \mathbf{r}=\delta_{s s^{\prime}}
$$

the following function:

$$
\mathcal{E}(\omega, \mathbf{r})=\sum_{s} \delta\left(\omega-\omega_{s}\right) \mathbf{e}(s, \mathbf{r})^{*} \cdot \mathbf{e}(s, \mathbf{r})
$$

describes the strength of the vacuum fluctuations of the electric field. Here, $\sum$ in general represents a summation over discrete modes and an integration over a continuum of modes. However, for the 2-D structures considered in this paper, there are no true discrete modes, and consequently $\sum$ refers to an integration in this paper. The details of how $\mathcal{E}(\omega, r)$ is calculated will be postponed until after (13) and (14). The function $\mathcal{E}$ will be referred to as the position-dependent photon density of states.

The following general expression for the rate of spontaneous emission from a two-level atom with transition frequency $\Omega$, and transition dipole moment parallel to the cartesian axis $j$, was given in [26]

$$
\Gamma_{j}=\frac{2 \pi}{\hbar^{2}}|M|^{2}\left(-\frac{1}{\pi} \operatorname{Imag}\left(G_{j j}^{R}(\mathbf{r}, \mathrm{r} ; \Omega)\right)\right) .
$$

Here, $M$ is the atomic matrix element or dipole matrix element, and $G_{i j}^{R}\left(\mathbf{r}, \mathbf{r}^{\prime} ; \omega\right)$ is the Fourier transform of the retarded Green's function of the transverse electric field.

The general approach using the Green's function (5) may be applied to structures with a complex dielectric function, i.e., absorbing dielectric media. Here, we will restrict ourselves to a real dielectric function, and we will also average the emission rate over all possible orientations for the transition dipole moment.

In this case, when the position-dependent photon density of states $\mathcal{E}$ is a smooth function in the frequency $\omega$, the rate of spontaneous emission may be related to the position-dependent 
photon density of states $\mathcal{E}(\omega, \mathrm{r})$ by the following expression [24], [27]:

$$
\Gamma=\frac{2 \pi}{\hbar^{2}}|M|^{2} \mathcal{E}(\Omega, \mathbf{r}) .
$$

For a homogeneous dielectric, the function $\mathcal{E}(\omega, \mathbf{r})$ becomes parabolic in $\omega$ and independent of the position $\mathbf{r}$, i.e., $\mathcal{E}_{\mathrm{hom}}(\omega)=\mathcal{E}(\omega, \mathrm{r})$. Consider a two-level atom located at position $\mathbf{r}$ in a homogeneous dielectric. By modifying the dielectric in a distance from the atom being large relative to the size of an atom, we expect $|M|^{2}$ to be unchanged. However, if this distance is not large relative to the emission wavelength of interest, $\mathcal{E}(\omega, r)$ may change significantly. In this case, the change in the rate of spontaneous radiative decay for the atom due to the change in the dielectric $\varepsilon_{r}(\mathbf{r})$ is given by the ratio

$$
\frac{\mathcal{E}(\Omega, \mathbf{r})}{\mathcal{E}_{\text {hom }}(\Omega)} .
$$

The evaluation of (7) for photonic crystals requires a calculation of the eigenfunctions $\mathbf{e}(s, \mathbf{r})$. We calculate these eigenfunctions using a numerical method based on plane-wave expansion theory, effective medium theory, and a variational principle [21], [22], [28], [29]. For the case of a real dielectric function, the magnetic-field wave equation

$$
\nabla \times\left(\frac{1}{\varepsilon_{r}(\mathbf{r})} \nabla \times \mathbf{H}(\mathbf{r})\right)=\frac{\omega^{2}}{c^{2}} \mathbf{H}(\mathbf{r})
$$

may be treated as a Hermitian eigenvalue problem, where H represents the eigenvector and $\omega^{2} / c^{2}$ is the corresponding eigenvalue.

The numerical approach used in this paper requires that the considered structures are characterized by discrete translational symmetry in the $x y$ plane. The simplest unit cell required for a representation of the photonic crystal with no defect is shown in Fig. 1(a). The photonic crystal with a microcavity introduced is approximated using a supercell approximation, i.e., the structure is approximated with the discrete-translationally symmetric structure described by the supercell shown in Fig. 1(b). For the case of structures characterized by discrete translational symmetry, a solution may, according to Bloch's theorem, be written in the form

$$
\mathbf{H}_{\mathbf{k}, n}(\mathbf{r})=\sum_{\mathbf{G}} \sum_{\lambda=1,2} \mathbf{h}_{\mathbf{k}, \mathbf{G}, \lambda, n} e^{i(\mathbf{k}+\mathbf{G}) \cdot \mathbf{r}}
$$

where $\mathbf{k}$ is a wavenumber vector, $\mathbf{G}$ is a reciprocal lattice vector, and $\lambda$ represents the two field directions perpendicular to $\mathbf{k}+\mathbf{G}$. The index $n$ is the band number. The eigenvalue corresponding to the eigenvector $\mathbf{H}_{\mathbf{k}, n}$ is denoted $\omega_{\mathbf{k}, n}^{2} / c^{2}$. The eigenfunctions (9) are found using a variational method based on minimization of the functional

$$
E(\mathbf{H})=\frac{\left\langle\nabla \times\left(\frac{1}{\varepsilon_{r}(\mathbf{r})} \nabla \times \mathbf{H}\right) \mid \mathbf{H}\right\rangle}{\langle\mathbf{H} \mid \mathbf{H}\rangle} .
$$

When this functional is at a minimum, the argument $\mathbf{H}$ is an eigenvector and $E(\mathbf{H})$ is the corresponding eigenvalue. By inserting a trial vector of the form (9) into (10), the functional effectively becomes a function of the coefficients $\mathbf{h}_{\mathbf{k}, \mathbf{G}, \lambda, n}$, and the problem is reduced to varying the coefficients along a path that minimizes the functional. An efficient iterative approach that performs this task is described in [28]. Higher order solutions are found by restricting the trial vectors to be orthogonal to all previously found eigenvectors and using the same minimization principle. The calculation time is reduced by performing the rotations $\nabla \times$ in Fourier space and the operation $1 / \varepsilon_{r}(\mathrm{r})$ in real space. The fast Fourier transform (FFT) is used to go from one space to the other. Using this approach, however, interpolation of the dielectric function in real space is necessary to achieve good convergence. A method for interpolation based on effective medium theory [29] and a tensor representation of the dielectric constant is described in [21], [22]. The required computer memory scales linearly with the number of plane waves $N$ used in the expansion (9), whereas the calculation time scales as $N \log (N)$. Therefore, relative to previous plane-wave methods [23], this approach offers a significant improvement for large numerical problems making accurate modeling of active photonic crystals with defects feasible.

The electric field in translationally symmetric dielectric structures may, in general, be written in the form

$$
\mathbf{E}(\mathbf{r}, t)=\operatorname{Real}\left(\sum_{\mathbf{k}, n} \mathbf{E}_{\mathbf{k}, n}(\mathbf{r}) e^{i \omega_{\mathbf{k}, n} t}\right)
$$

where the complex field distribution $\mathbf{E}_{\mathbf{k}, n}(\mathbf{r})$ represents the $n$th complex eigensolution related to the wavenumber vector k. These field distributions for the electric field are derived from the numerically calculated solutions for the magnetic field using the usual relation

$$
\mathbf{E}_{\mathbf{k}, n}(\mathbf{r})=\frac{1}{i \omega_{\mathbf{k}, n} \varepsilon_{r}(\mathbf{r})} \nabla \times \mathbf{H}_{\mathbf{k}, n}(\mathbf{r}) .
$$

With the formulation (11), the position-dependent density of states (4) may be written

$$
\mathcal{E}(\omega, \mathbf{r})=\sum_{\mathbf{k}, n} \delta\left(\omega-\omega_{\mathbf{k}, n}\right)\left|\mathbf{E}_{\mathbf{k}, n}(\mathbf{r})\right|^{2} .
$$

The usual density of states may, correspondingly, be written

$$
\rho(\omega)=\sum_{\mathbf{k}, n} \delta\left(\omega-\omega_{\mathbf{k}, n}\right) .
$$

In-plane photonic bandgaps can be defined using the in-plane density of states, which correspond to (14), where only the $\mathrm{k}$-vectors with no component normal to the $x y$ plane are summed over, i.e., $k_{z}=0$. Previous calculations of the position-independent in-plane photon density of states may be found in [30].

In (13) and (14), the in-plane component of $\mathbf{k}$ should be integrated over the first Brillouin zone, and the out-of-plane component of $\mathbf{k}$ should be integrated over an interval large enough to ensure that the frequency interval of interest is covered. The integration is performed numerically by sampling $\mathbf{k}$ at a large number of discrete equidistant points in reciprocal space. The density of states (14) is then obtained by counting the number of $\omega_{\mathbf{k}, n}$ corresponding to the discrete values of $\mathbf{k}$ that fall within 


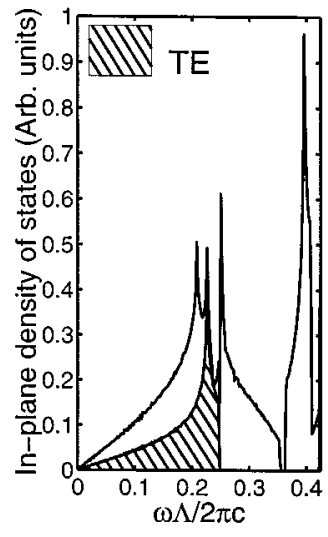

(a)

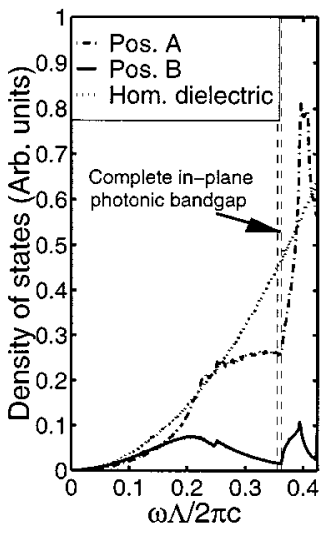

(b)
Fig. 2. (a) Position-independent in-plane density of states for the triangular-hole photonic crystal shown in Fig. 1(a). (b) Position-dependent density of states for two positions A (dash-dot line) and B (solid line) in the same structure. Also shown is the density of states for a homogeneous dielectric with the same dielectric constant as the background material (dotted line). The upper and lower edges of the complete in-plane photonic bandgap are shown with two dashed lines.

small frequency intervals. Furthermore, the position-dependent density of states (13) for a given position $\mathbf{r}$ is obtained by weighting the contribution from each state by the normalized amplitude of the electric field squared.

It should be noted that the integration over the first Brillouin zone in (13) and (14) may be simplified significantly for dielectric structures characterized by various symmetries. In our case, all field solutions may be derived from the field solutions related to $1 / 12$ of the first Brillouin zone, i.e., the irreducible first Brillouin zone. Furthermore, the Fourier coefficients in (9) may be restricted to be real due to the inversion symmetry of the considered structures.

\section{RESUlTS AND DisCUSSION}

In this section, numerical results will be given for the position-dependent photon density of states and the rate of spontaneous radiative decay for the photonic crystal and photonic crystal microcavity shown in Fig. 1. The properties of the radiation field in the photonic crystal with no microcavity introduced [see Fig. 1(a)] are summarized in Fig. 2. The in-plane density of states shown to the left in Fig. 2 illustrates the in-plane photonic bandgap properties of the photonic crystal. An in-plane photonic bandgap is defined as a frequency interval where the in-plane photon density of states is zero. Fig. 2 shows that in-plane propagating modes with TE polarization (magnetic field parallel to the $z$ axis) are not allowed within the frequency interval from $\omega \Lambda / 2 \pi c=0.25$ to 0.42 , where $\omega$ is the angular frequency, $\Lambda$ is the center-to-center hole spacing defined in Fig. 1(a), and $c$ is the speed of light in vacuum. This frequency interval will be referred to as a TE bandgap. Fig. 2 also shows the complete in-plane density of states for the photonic crystal, taking into account both TE and TM polarized modes. TM refers to modes with the electric field parallel to the $z$ axis. A TM bandgap exists from the frequency $\omega \Lambda / 2 \pi c=0.355$ to 0.362 . In fact, the frequency interval from $\omega \Lambda / 2 \pi c=0.355$ to 0.362 represents a

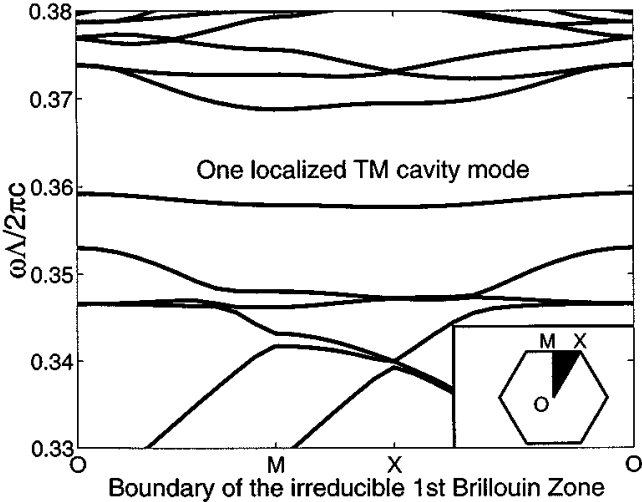

Fig. 3. In-plane band diagram of TM-polarized modes for the supercell shown in Fig. 1 taken over the boundary of the irreducible first Brillouin zone. The inset shows the first Brillouin zone, and the black region within this zone corresponds to the irreducible first Brillouin zone.

complete in-plane photonic bandgap since the TE bandgap and the TM bandgap overlap.

The in-plane photonic bandgaps related to either TE or TM polarization are important for understanding localization of electromagnetic modes to defects in the photonic crystal. TE (TM) polarized cavity modes in the photonic crystal microcavity shown in Fig. 1(b) must have a frequency within the corresponding TE (TM) photonic bandgap.

Along with the in-plane density of states, Fig. 2 shows the position-dependent density of states equation (13) midway between two air holes (Pos. A) and in the center of an air hole (Pos. B). The two positions A and B are shown in Fig. 1(a). Along with the position-dependent density of states for the photonic crystal, a similar calculation is shown for a homogeneous dielectric with the same dielectric constant as the photonic crystal background material. For an atom with a transition frequency within the complete in-plane photonic bandgap, it is clear according to (7) that, by taking the ratio between the curve for position A and the curve for a homogeneous dielectric, the rate of spontaneous emission midway between two air holes is suppressed by $42 \%$ relative to the corresponding rate in the homogeneous dielectric. The position-dependent density of states at the center of an air hole (Pos. B) is shown to illustrate that the position-dependent density of states depends strongly on position. The density-of-states calculations in Fig. 2 were calculated using $32 \times 32$ reciprocal lattice vectors $\mathbf{G}$ in (9). The position-dependent density of states equation (13) was calculated using approximately 840000 reciprocal space points $\mathbf{k}$, whereas approximately 130000 points were used for the in-plane density of states.

As a cavity is created in the photonic crystal by removing an air hole in the periodic structure, electromagnetic modes may exist being localized to the cavity. These modes are interesting as lasing modes in future lasers based on photonic crystals.

Fig. 3 shows a band diagram taken over the boundary of the irreducible first Brillouin zone for TM-polarized modes related to the periodic structure defined by the supercell shown in Fig. 1(b). The inset shows the first Brillouin zone, and the black region within this zone corresponds to the irreducible first Brillouin zone. In the band diagram, one defect band exists within 


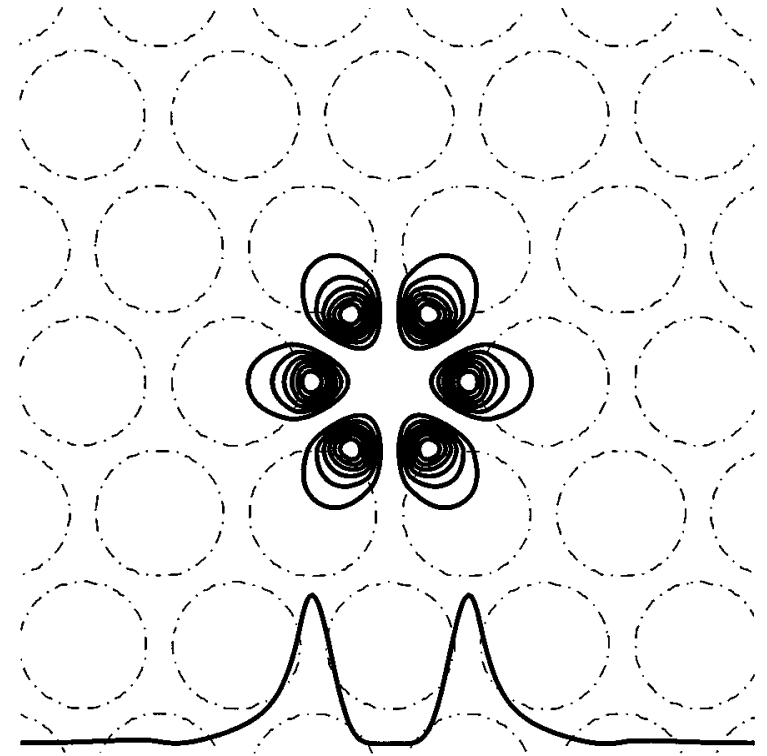

Fig. 4. Amplitude of the electric field squared for a nondegenerate TM-polarized cavity mode with normalized frequency $\omega \Lambda / 2 \pi c=0.36$.

the complete in-plane photonic bandgap from $\omega \Lambda / 2 \pi c=0.355$ to 0.362 for the photonic crystal with no microcavity introduced. This band corresponds to a nondegenerate TM-polarized mode being localized to the photonic crystal microcavity. Note that, due to the finite size of the supercell, the upper edge of the bandgap is shifted toward higher frequencies. For this periodic structure, the microcavity is repeated periodically, and the distance between neighboring microcavities is five photonic crystal lattice constants $\Lambda$. Due to the finite distance between neighboring cavities, we see a broadening of the defect band. This broadening is due to coupling between neighboring cavities. As the distance between neighboring cavities is increased, the broadening of the defect band will eventually decrease to zero, and in this limit the defect band gives rise to a delta function peak in the in-plane density of states. However, for any finite distance between neighboring cavities, i.e., any finite size supercell, the defect band will be broadened.

The band diagram in Fig. 3 was calculated for a zero out-ofplane component of $\mathbf{k}$, i.e., $k_{z}=0$. However, as $k_{z}$ is increased, the bands will also increase in frequency [9], [16], [17], [31], and a continuum of modes is formed. Consequently, a delta function peak will not appear in the density of states given by (13) and (14). Since the integration in (13) and (14) is over a continuum of modes, the approach of dividing the frequency axis into small intervals and considering only a finite number of discrete vectors $\mathbf{k}$ is justified.

The amplitude of the electric field squared for the nondegenerate TM-polarized cavity mode with normalized frequency $\omega^{\prime} \Lambda / 2 \pi c=0.36$ is shown in Fig. 4. The dashed-dotted lines represent the air holes in the photonic crystal, whereas the solid curves in the region of the microcavity are contour lines of the amplitude of the electric field squared. Below the cavity is shown a horizontal cross section of the amplitude squared through the center of the cavity. Note that, for TM polarization. the electric field is parallel to the interfaces between the

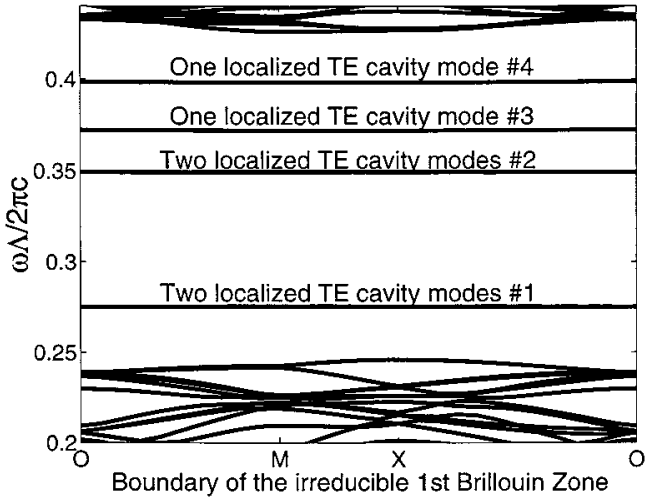

Fig. 5. In-plane band diagram of TE-polarized modes for the supercell shown in Fig. 1.

air regions and the high-index material regions in the structure. Therefore, the electric field is continuous across these interfaces. This does not have to be the case for TE-polarized modes, where the electric field may have a component perpendicular to the interface.

The amplitude of the TM-polarized mode is high at position C and low at position $\mathrm{D}$. Therefore, for the frequency $\omega^{\prime} \Lambda / 2 \pi c=$ 0.36 , a strong peak is expected in the position-dependent density of states (13) at position C, whereas a peak is not expected at position $\mathrm{D}$. The peak at position $\mathrm{C}$ is expected to start abruptly at the frequency $\omega^{\prime} \Lambda / 2 \pi c=0.36$ and is expected to roll off roughly as $1 / \sqrt{\left(\omega^{2}-\omega^{\prime 2}\right)}$, as was the case for a metallic wire waveguide in [32]. This behavior is similar to the behavior of the electronic density of states in an electronic quantum wire. In fact, we may think of 2-D photonic crystal microcavities as the optical analog to electronic quantum wires. Due to the broadening of the defect band, the calculated density of states using a finite size supercell is not expected to be singular at the frequency $\omega^{\prime} \Lambda / 2 \pi c=0.36$. However, if the size of the supercell is increased toward infinity, the calculated density of states at this frequency may eventually become singular.

A band diagram for the TE-polarized modes related to the periodic structure defined by the supercell shown in Fig. 1(b) is shown in Fig. 5. In this case, we find two degenerate bands at the frequency $\omega \Lambda / 2 \pi c=0.27$. Two degenerate bands are also found at the frequency $\omega \Lambda / 2 \pi c=0.35$, and nondegenerate bands are found at the frequencies $\omega \Lambda / 2 \pi c=0.37$ and $\omega \Lambda / 2 \pi c=0.40$. These defect bands are all within the TE bandgap and correspond to TE-polarized modes being localized to the 2-D microcavity. Although these bands appear to be flat, they are, in fact, slightly broadened due to the finite size of the supercell.

The two bands at the frequency $\omega \Lambda / 2 \pi c=0.27$ correspond to two degenerate orthogonal cavity modes. There are, however, many possible ways of choosing the two orthogonal TE modes. Other choices of modes may be obtained by making linear combinations of the two modes. In general, the amplitude of the electric field squared for one of these two modes does not exhibit $60^{\circ}$ rotational symmetry about the center of the microcavity. However, if the two orthogonal degenerate modes are normalized to unity energy within the supercell, then the sum of 


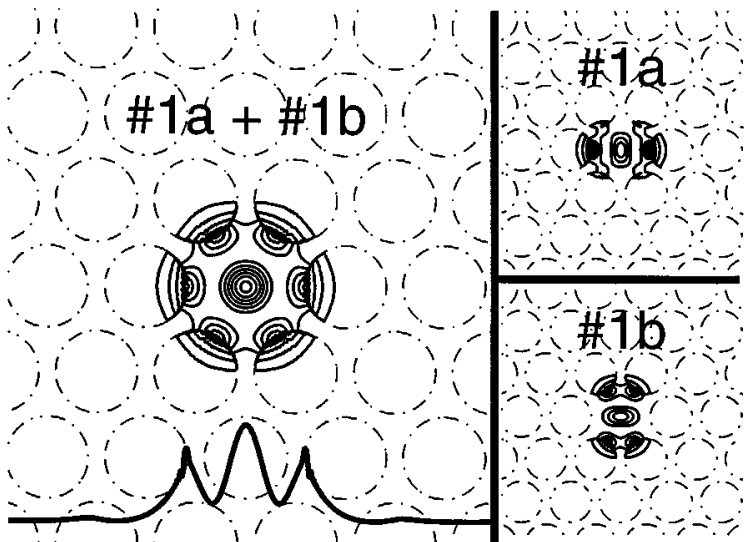

Fig. 6. Amplitude of the electric field squared for one possible choice for two orthogonal degenerate TE-polarized cavity modes \#1a and \#1b with normalized frequency $\omega \Lambda / 2 \pi c=0.27$. Also shown is the sum of the amplitudes squared for the two modes.

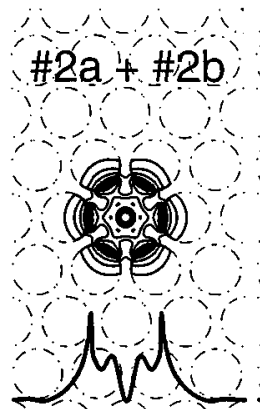

(a)

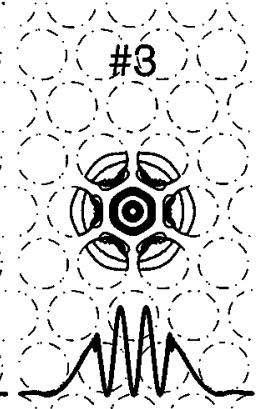

(b)

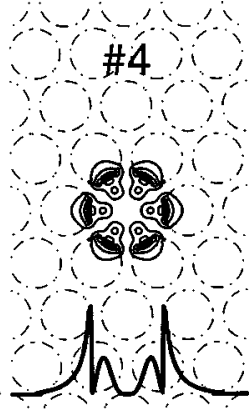

(c)
Fig. 7. (a) The sum of the amplitudes of the electric field squared for two orthogonal, degenerate and normalized TE-polarized cavity modes \#2a and \#2b with normalized frequency $\omega \Lambda / 2 \pi c=0.35$. (b) Amplitude of the electric field squared for a TE-polarized cavity mode \#3 with frequency $\omega \Lambda / 2 \pi c=0.37$. (c) Amplitude of the electric field squared for a TE-polarized cavity mode \#4 with frequency $\omega \Lambda / 2 \pi c=0.40$.

the electric field squared for the two modes does not depend on how these modes were chosen. From symmetry considerations, the sum must exhibit $60^{\circ}$ rotational symmetry about the center of the microcavity. Furthermore, according to (4), it is indeed the sum of the amplitudes of the electric field squared which is the relevant parameter in a calculation of the position-dependent photon density of states. The amplitude of the electric field squared is shown for one possible choice of two orthogonal degenerate modes denoted \#1a and \#1b in Fig. 6. Also shown is the more important sum of the amplitudes of the electric field squared for the two modes. The summation of the intensities peaks at the center of the microcavity [position D in Fig. 1(b)]. The intensity is also high near the air holes surrounding the microcavity [position $\mathrm{C}$ in Fig. 1(b)]. Therefore, for the frequency $\omega \Lambda / 2 \pi c=0.27$, a high peak is expected at position $\mathrm{D}$ for the position-dependent photon density of states, and a smaller peak is expected at position $\mathrm{C}$.

Fig. 7 shows the amplitude of the electric field squared for the other TE-polarized cavity modes denoted \#2, \#3, and \#4 in the band diagram. For the two degenerate modes denoted \#2, only the sum of the amplitudes squared is shown. We should note that the frequency of the TE-polarized cavity modes \#2
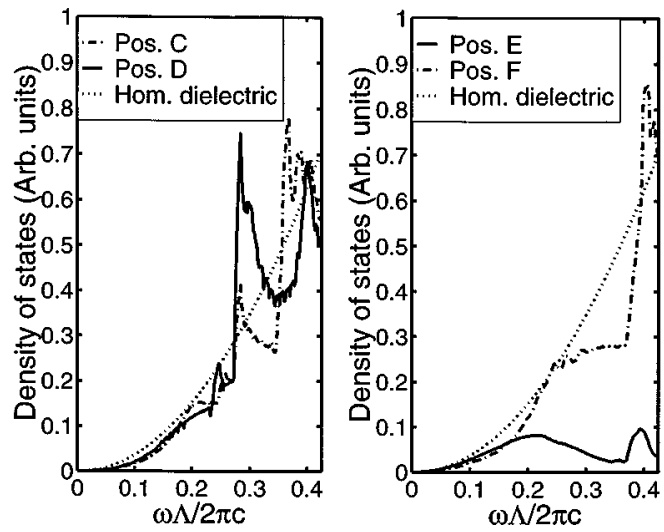

Fig. 8. Position-dependent density of states for four positions C, D, E, and F in the structure shown in Fig. 1(b).

$(\omega \Lambda / 2 \pi c=0.35)$ is close to the frequency of the TM-polarized cavity mode $(\omega \Lambda / 2 \pi c=0.36)$.

In Fig. 8, we have shown the position-dependent density of states for the four positions labeled C, D, E and F in Fig. 1(b). The number of eigenvectors $\mathbf{E}_{\mathbf{k}, n}$ used for calculating Fig. 8 is equivalent to the number of eigenvectors used for calculating Fig. 2. However, for the calculation of Fig. 8, we used $128 \times 128$ reciprocal lattice vectors $\mathbf{G}$ in (9).

The two positions $\mathrm{E}$ and $\mathrm{F}$ in Fig. 1(b) are similar to the positions $\mathrm{A}$ and $\mathrm{B}$ in Fig. 1(a) in the sense that A and F are both located midway between two air holes, whereas B and E are both located at the center of an air-hole. By comparing Fig. 8 and Fig. 2, it is clear that the difference in the density of states in the two cases differs only slightly. As a result, the position-dependent density of states and the rate of spontaneous radiative decay, in a distance of a few photonic crystal lattice constants $\Lambda$ from the defect, is only weakly affected by the presence of the defect.

For a photonic crystal microcavity laser, the active medium must be placed in such a way that it interacts with a cavity mode. Therefore, the active medium must be placed at the positions where the intensity of the relevant cavity mode is high. Two relevant positions for the TE-polarized cavity modes shown in Fig. 6 are the positions $C$ and $D$, whereas position $C$ is relevant for the TM-polarized cavity mode shown in Fig. 4.

The position-dependent density of states at positions $\mathrm{C}$ and $\mathrm{D}$ is shown to the left in Fig. 8. A clear peak is seen in the density of states at the center of the cavity (Pos. D) for the frequency $\omega \Lambda / 2 \pi c=0.27$, whereas a smaller peak is seen near the edge of the cavity (Pos. C). This is in agreement with the intensity profiles of the TE-polarized modes shown in Fig. 6.

The peak at position $\mathrm{C}$ near the frequencies $\omega \Lambda / 2 \pi c=0.35$ and $\omega \Lambda / 2 \pi c=0.36$ may in a similar way be related to the TE-polarized cavity modes \#2 and the TM-polarized cavity mode. Two peaks could have been expected. However, if the first peak rolls off slowly and is broadened, then the result is that only one peak is seen.

For the frequency $\omega \Lambda / 2 \pi c=0.4$, another peak is seen in the density of states at position D. Even though a TE-polarized cavity mode (\#4) exists at this frequency, the amplitude of the electric field squared for this mode is practically zero at position D. The peaks related to cavity modes are characterized by a very 
steep edge at the frequency of the cavity modes. This steep edge is seen for the frequencies 0.27 (TE-polarized cavity modes \#1) and 0.36 (TM-polarized cavity mode). A steep edge is, however, not seen for the peak at the frequency 0.4 at position $\mathrm{D}$, and this peak cannot be related to a cavity mode. Similarly, the TE-polarized cavity mode (\#3) with frequency 0.37 does not give rise to a peak at position $\mathrm{D}$ at this frequency. However, a small peak is seen at position $\mathrm{C}$ for this frequency.

By comparison with the density of states for a homogeneous dielectric, it is clear that the rate of spontaneous radiative decay may be enhanced relative to a homogeneous dielectric. This enhancement is due to the strongly localized cavity modes. For the case of a narrow linewidth atomic source at position $\mathbf{r}$ and transition frequency $\Omega$, the spontaneous emission factor $\beta$, i.e., the fraction of the total spontaneous emission going into a cavity mode $\mathbf{E}_{\mathrm{cav}}(\omega, \mathbf{r})$, is given by

$$
\beta(\Omega, \mathbf{r})=\frac{\left|\mathbf{E}_{\mathrm{cav}}(\Omega, \mathbf{r})\right|^{2}}{\mathcal{E}(\Omega, \mathbf{r})} .
$$

This expression for $\beta$ is similar to (3) in [33]. For a given atomic transition frequency $\Omega$, the denominator in the expression for $\beta$ at positions $\mathrm{C}$ and $\mathrm{D}$ may be obtained from Fig. 8. For the frequency $\omega \Lambda / 2 \pi c=0.27$, the nominator is then the contribution to the density of states from the TE-polarized cavity modes \#1. This contribution is identified as the steep edge at this frequency. For an atom at position D with transition frequency $\omega \Lambda / 2 \pi c=0.27$, this leads to a conservative estimate of $\beta \approx 70 \%$. The estimated spontaneous emission factor may increase as the distance between neighboring cavities, i.e., the size of the supercell, increases. This is expected since the broadening of the defect bands \#1 decreases as the size of the supercell increases.

Using the same approach for an atom at position $\mathrm{C}$ with transition frequency 0.36 , we would expect a large fraction of the spontaneous emission to go into the TM-polarized cavity mode shown in Fig. 4. However, another large fraction of the emission may also go into modes similar to the TE-polarized cavity modes \#2 with a nonzero out-of-plane component of the wavevector $\mathbf{k}$.

\section{CONCLUSIONS}

It has been shown that spontaneous emission may be suppressed by at least $42 \%$ for an electric dipole with random orientation in a 2-D photonic crystal.

By comparing the position-dependent photon density of states at two positions within the photonic crystal, it was clear that the strength of the electric field vacuum fluctuations depends strongly on position. Furthermore, the rate of spontaneous emission in the photonic crystal is practically unaffected by the presence of a defect or a microcavity being only a few photonic crystal lattice constants away. Strong peaks in the position-dependent density of states at two positions within a photonic crystal microcavity have been related to a few strongly localized cavity modes. The rate of spontaneous radiative decay for a narrow-linewidth two-level atom inside the photonic crystal microcavity interacting with cavity modes may be significantly enhanced. However, in this case, a large fraction of the spontaneous emission, at least $70 \%$, may go into the cavity modes.

\section{REFERENCES}

[1] E. Yablonovitch, "Inhibited spontaneous emission in solid-state physics and electronics," Phys. Rev. Lett., vol. 58, no. 20, pp. 2059-2062, May 1987.

[2] E. M. Purcell, "Spontaneous emission probabilities at radio frequencies," Phys. Rev., vol. 69, p. 681, 1946.

[3] R. G. Hulet and E. S. Hilfer, "Inhibited spontaneous emission by a rydberg atom," Phys. Rev. Lett., vol. 55, no. 20, pp. 2137-2140, Nov. 1985.

[4] R. K. Chang and A. J. Campillo, Optical Processes in Microcavities, Singapore, 1996. World Scientific.

[5] S. John, "Localization of light: Theory of photonic band gap materials," in Photonic Band Gap Materials. ser. E, C. M. Soukoulis, Ed. Dordrecht, The Netherlands: Kluwer, 1996, pp. 563-666. vol. of 315 NATO ASI Series, Applied sciences.

[6] W. Lukosz, "Theory of optical-environment-dependent spontaneous-emission rates for emitters in thin layers," Phys. Rev. B, vol. 22, no. 6, pp. 3030-3038, Sept. 1980.

[7] M. R. Philpott, "Fluorescence from molecules between mirrors," Chem. Phys. Lett., vol. 19, no. 3, pp. 435-439, Apr. 1973.

[8] P. W. Milonni and P. L. Knight, "Spontaneous emission between mirrors," Opt. Commun., vol. 9, no. 2, pp. 119-122, Oct. 1973.

[9] J. D. Joannopoulos, J. N. Winn, and R. D. Meade, Photonic Crystals: Molding the Flow of Light. Princeton, NJ: Princeton University Press, 1995.

[10] E. Yablonovitch, "Photonic bandgap structures," J. Opt. Soc. Amer. B, vol. 10, p. 283, 1993.

[11] J. G. Fleming and S.-Y. Lin, "Three-dimensional photonic crystal with a stop band from 1.35 to $1.95 \mu \mathrm{m}$," Opt. Lett., vol. 24, no. 1, pp. 49-51, Jan. 1999.

[12] S. Noda, N. Yamamoto, H. Kobayashi, M. Okano, and K. Tomoda, "Optical properties of three-dimensional photonic crystals based on iii-v semiconductors at infrared to near-infrared wavelengths," Appl. Phys. Lett., vol. 75, no. 7, pp. 905-907, Aug. 1999.

[13] O. Painter, J. Vuckovic, and A. Scherer, "Defect modes of a two-dimensional photonic crystal in an optically thin dielectric slab," J. Opt. Soc. Amer. B, vol. 16, no. 2, pp. 275-285, 1999.

[14] O. Painter, R. K. Lee, A. Yariv, and A. Scherer, "Two-dimensional photonic bandgap defect laser," in CLEO'99, May 23-28, 1999, paper CPD21.

[15] O. Painter, R. K. Lee, A. Scherer, A. Yariv, J. D. O'Brien, P. D. Dapkus, and I. Kim, "Two-dimensional photonic band-gap defect mode laser," Science-AAAS-Weekly Paper Edition, vol. 284, no. 5421, pp. 1819-1821, 1999.

[16] X. P. Feng and Y. Arakawa, "Off-plane angle dependence of photonic band gap in a two-dimensional photonic crystal," IEEE J. Quantum Electron., vol. 32, pp. 535-542, Mar. 1996.

[17] T. Søndergaard, J. Broeng, A. Bjarklev, K. Dridi, and S. E. Barkou, "Suppression of spontaneous emission for a two-dimensional honeycomb photonic bandgap structure estimated using a new effective-index model," IEEE J. Quantum Electron., vol. 34, pp. 2308-2313, Dec. 1998.

[18] R. Loudon, The Quantum Theory of Light, 2nd ed. Oxford, U.K.: Clarendon Press, 1983.

[19] T. Søndergaard, J. Broeng, and A. Bjarklev, "Suppression of spontaneous emission for two-dimensional GaAs photonic crystal microcavities," in $C L E O^{\prime} 99$, May 23-28, 1999, paper JFA2.

[20] K. Busch and S. John, "Photonic band gap formation in certain selforganizing systems," Phys. Rev. E, vol. 58, no. 3, pp. 3896-3908, Sept. 1998.

[21] R. D. Meade, A. M. Rappe, K. D. Brommer, J. D. Joannopoulos, and O. L. Alerhand, "Accurate theoretical analysis of photonic band-gap materials," Phys. Rev. B, vol. 48, no. 11, pp. 8434-8437, Sept. 1993.

[22] — "Erratum: Accurate theoretical analysis of photonic band-gap materials," Phys. Rev. B, vol. 55, no. 23, p. 15 942, June 1997.

[23] K. M. Ho, C. T. Chan, and C. M. Soukoulis, "Existence of a photonic gap in periodic dielectric structures," Phys. Rev. Lett., vol. 65, no. 25, pp. 3152-3155, Dec. 1990

[24] S. C. Ching, H. M. Lai, and K. Young, "Dielectric microspheres as optical cavities: Einstein a and b coefficients and level shift," J. Opt. Soc. Amer. B, vol. 4, no. 12, pp. 2004-2009, Dec. 1987.

[25] S. Ramo, J. R. Whinnery, and T. van Ducer, Fields and Waves in Communication Electronics, 3rd ed. New York, NY: Wiley, 1994. 
[26] S. M. Barnett, B. Huttner, and R. Loudon, "Spontaneous emission in absorbing dielectric media," Phys. Rev. Lett., vol. 68, no. 25, pp. 3698-3701, June 1992

[27] E. S. C. Ching, P. T. Leung, and K. Young, "The role of quasinormal modes," in Optical Processes in Microcavities, R. K. Chang and A. J. Campillo, Eds, Singapore: World Scientific, 1996.

[28] M. P. Teter, M. C. Payne, and D. C. Allan, "Solution of Schrödinger's equation for large systems," Phys. Rev. B, vol. 40, no. 18, pp 12255-12263, Dec. 1989.

[29] D. E. Aspnes, "Local-field effects and effective-medium theory: A microscopic perspective," Amer. J. Phys., vol. 50, no. 8, pp. 704-709, Aug. 1981.

[30] M. Plihal and A. A. Maradudin, "Photonic band structure of two-dimensional systems," Phys. Rev. B, vol. 44, no. 16, p. 8565, Oct. 1991.

[31] A. A. Maradudin and A. R. McGurn, "Out of plane propagation of electromagnetic waves in a two-dimensional periodic dielectric medium," $J$. Mod. Opt., vol. 41, no. 2, pp. 275-284, 1994.

[32] S. D. Brorson, H. Yokoyama, and E. P. Ippen, "Spontaneous emission rate alteration in optical waveguide structures," IEEE J. Quantum Electron., vol. 26, pp. 1492-1499, Sept. 1990
[33] M. P. van Exter, G. Nienhuis, and J. P. Woerdman, "Two simple expressions for the spontaneous emission factor $\beta$," Phys. Rev. A, vol. 54, no. 4, pp. 3553-3558, Oct. 1996.

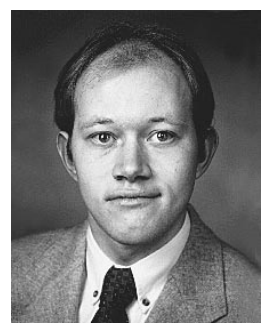

Thomas Søndergaard received the M.Sc.E.E degree from the Technical University of Denmark, Lyngby, in March 1999. He is currently pursuing the Ph.D. degree in electrical engineering at Research Center COM at the Technical University of Denmark, where his work involves modeling of interaction between matter and light in active structures.

Previous work includes modeling of erbium-doped fiber lasers and planar waveguide lasers with Bragg gratings, photonic crystal fibers, and spontaneous emission rate alteration in photonic crystals. 